\title{
The Influence of Maggot Excretions on PAO1 Biofilm Formation on Different Biomaterials
}

\author{
Gwendolyn Cazander MD, Kiril E. B. van Veen, \\ Lee H. Bouwman MD, PhD, Alexandra T. Bernards MD, PhD, \\ Gerrolt N. Jukema MD, PhD
}

Received: 13 November 2007 / Accepted: 17 September 2008/Published online: 16 October 2008

(C) The Association of Bone and Joint Surgeons 2008

\begin{abstract}
Biofilm formation in wounds and on biomaterials is increasingly recognized as a problem. It therefore is important to focus on new strategies for eradicating severe biofilm-associated infections. The beneficial effects of maggots (Lucilia sericata) in wounds have been known for centuries. We hypothesized sterile maggot excretions and secretions (ES) could prevent, inhibit, and break down biofilms of Pseudomonas aeruginosa (PAO1) on different biomaterials. Therefore, we investigated biofilm formation on polyethylene, titanium, and stainless steel. Furthermore, we compared the biofilm reduction capacity of Instar-1 and Instar-3 maggot ES and tested the temperature tolerance of ES. After biofilms formed in M63 nutrient medium on comb-forming models of the biomaterials, ES solutions in phosphate-buffered saline or M63 were added in different concentrations. PAO1 biofilms adhered tightly to polyethylene and titanium but weakly to stainless steel. Maggot ES prevent and inhibit PAO1 biofilm formation and even break down existing biofilms. ES still had considerable biofilm
\end{abstract}

One or more of the authors (GC, GNJ) have received funding from an unrestricted research grant of Kinetic Concepts Inc, Europe Holding BV, Amstelveen, The Netherlands.

G. Cazander, K. E. B. van Veen, G. N. Jukema ( $₫)$ Department of Trauma Surgery, VU University Medical Center, PO Box 7057, 1007 MB Amsterdam, The Netherlands e-mail: g.jukema@vumc.nl

G. Cazander, K. E. B. van Veen, L. H. Bouwman Section of Traumatology, Department of Surgery, Leiden University Medical Center, Leiden, The Netherlands

A. T. Bernards

Department of Medical Microbiology, Leiden University

Medical Center, Leiden, The Netherlands reduction properties after storage at room temperature for 1 month. ES from Instar-3 maggots were more effective than ES from Instar-1 maggots. These results may be relevant to patient care as biofilms complicate the treatment of infections associated with orthopaedic implants.

\section{Introduction}

Biofilm formation (BF) on biomaterials is a major problem in trauma and orthopaedic surgery [6]. Bacteria adhering to prosthetic material can form a biofilm composed of a complex extracellular polysaccharide matrix in which they then become embedded [5]. The matrix prevents antibiotic penetration and as a result protects bacteria against antibiotics [7, 28]. Once infected, the implant often must be removed [18, 32]. Temporary implantation of antibiotic beads is sometimes necessary [19].

The importance of $\mathrm{BF}$ was not acknowledged until 30 years ago, when the successful effects of penicillin and other antibiotics were complicated by the increasing antibiotic resistance of bacteria [15]. In 1931, William Baer, an orthopaedic surgeon at Johns Hopkins Hospital in Baltimore, introduced maggot débridement therapy (MDT) [1] around the same time Fleming discovered penicillin, but MDT was supplanted because of the discovery of antibiotics [34]. Baer successfully used MDT for treatment of children with severe osteomyelitis [1]. Interest in MDT for wound healing was renewed in the 1980s [25], because the antibiotic resistance of bacteria increased rapidly [26] and antibiotic therapy and surgical treatment of wounds did not suffice in some cases. Currently, maggots of Lucilia sericata are widely used, have successful healing effects, and were approved by the US Food and Drug Administration in 
2004 (510[k] \#33391) [24, 27, 35]. Research to discover the underlying mechanisms of action by which maggots reduce bacterial infections could provide us with new treatment possibilities for severe, infected wounds.

For certain wounds, we use sterile maggots captured in small permeable bags, which consist of a 2- to 3-mm thin foam layer of polyvinyl alcohol. These bags allow free exchange of maggot excretions and secretions [11]. We have observed resolution of infections suspected for biofilm formation using maggots in bags. Therefore, we suspected maggot excretions and secretions (ES) could interfere with the biofilm in an infected wound and aid in healing. If maggot ES can reduce biofilms, there may be pharmacologic agents that could be developed from ES, which could provide new treatment methods for biofilmassociating infections.

We therefore asked whether (1) sterile maggot ES would break down biofilms, prevent biofilm formation, and inhibit further growth of existing biofilms; (2) the quantity of biofilm formation of Pseudomonas aeruginosa depended on the material surface (specifically, polyethylene, titanium, or surgical stainless steel); (3) Instar-1 and Instar-3 maggot ES comparably reduced biofilms; and (4) storage of ES under different conditions and temperature reduced the effects of ES on biofilms.

\section{Materials and Methods}

Biofilms were formed on comb-forming models of polyethylene (PE), titanium (TI), and surgical stainless steel (SSS) hanging in a 96-well microtiter plate with culture medium and bacteria. Either ES were added directly to the bacterial suspension to test biofilm prevention or ES were added after 24 or 48 hours of $\mathrm{BF}$ to investigate biofilm inhibition and/or breakdown. We used ES from Instar-1 and Instar-3 maggots. Temperature tolerance was tested by storing $\mathrm{ES}$ at $-80^{\circ} \mathrm{C}$, at $+4^{\circ} \mathrm{C}$, and at room temperature for 1 day, 1 week, and 1 month before using them.

We tested BF on comb-like devices consisting of eight prongs (produced according to our design and requested by Litos $\mathrm{GmbH}$, Hamburg, Germany). The prongs were made to fit into a flat-bottomed 96-well microtiter plate (Greiner Bio-One, Alphen a/d Rijn, The Netherlands) leaving $2 \mathrm{~mm}$ of free space at the sides and hanging $1 \mathrm{~mm}$ from the bottom of the well (Fig. 1). Combs were made of PE, TI, and SSS according to specifications for common patient implants. All combs were sterilized by low-temperature hydrogen peroxide gas plasma sterilization. We considered each prong to be one test, and assays were performed in at least quadruplicate. All controls were made in octuplicate.

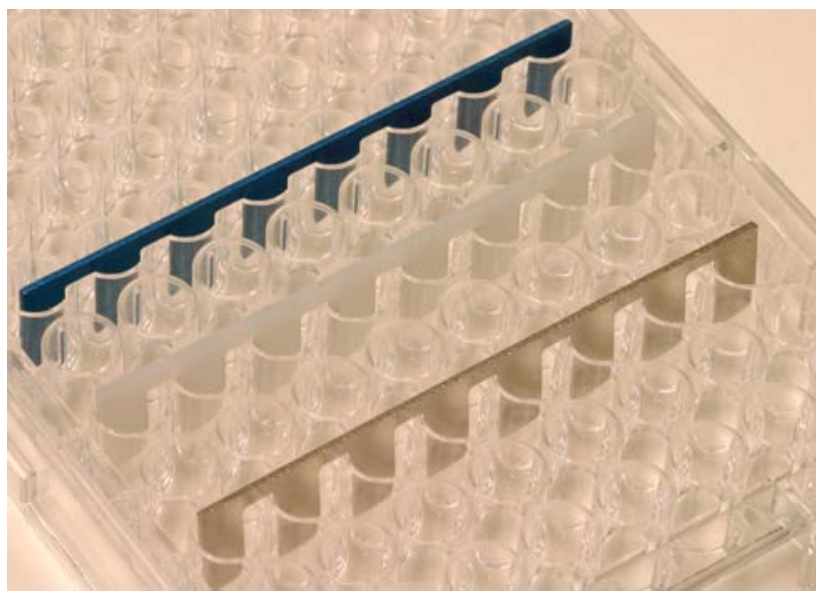

Fig. 1 Combs were made of TI (blue), PE (white), and SSS (silver) hanging in a 96-well microtiter plate. $\mathrm{TI}=$ titanium; $\mathrm{PE}=$ polyethylene; SSS = surgical stainless steel.

For our experiments, we chose P. aeruginosa strain PAO1 because it is one of the major causes of orthopaedic infections $[8,29]$ and it is able to form very stable biofilms [20]. Bacteria (ATCC 15692) were grown overnight in King's medium B [14] at $28^{\circ} \mathrm{C}$ under vigorous shaking (190 rpm). We prepared a solution of a stationary phase culture in phosphate-buffered saline (PBS) to a density of McFarland 0.5 corresponding with $1.5 \times 10^{8}$ colonyforming units (CFU) per milliliter. To determine the actual inoculum, viable counts were made by inoculating nutrient agar plates (Biomérieux, Lyon, France) with $10-\mu \mathrm{L}$ volumes of serial 1:10 dilutions of the suspension. We incubated the plates overnight at $37^{\circ} \mathrm{C}$ and the colonies were counted.

Biofilms were made in M63 nutrient medium, which allows for BF as previously described by O'Toole and Kolter [21]. We diluted the PAO1 suspension 1:30 in PBS and then 1:20 in M63. A volume of $100 \mu \mathrm{L}$ of the bacterial suspension was added to 24 wells of a sterile 96-well flatbottomed microtiter plate. The final inoculum in each well was $2.5 \times 10^{5} \mathrm{CFU} / \mathrm{mL}$. We placed the combs in the wells and incubated them for 24 hours at $37^{\circ} \mathrm{C}$, allowing for BF. Control combs were placed in M63 medium. Microtiter plates were covered using parafilm (Pechiney Plastic Packaging, Chicago, IL).

After 24 hours, we washed each comb by placing it under slow-running distilled tap water for 30 seconds. All combs were placed in a new 96-well plate, dried in a laminar flow cabinet for 5 minutes, and stained with $1 \%$ crystal violet for 15 minutes (Fig. 2). Excess stain was rinsed off by distilled water. After drying again in the flow cabinet, we suspended each comb in a new column of wells filled with $270 \mu \mathrm{L}$ ethanol absolute to absorb the crystal violet. To prevent evaporation of ethanol, plates were sealed with adhesive coverslips (Nutacon BV, Leimuiden, 


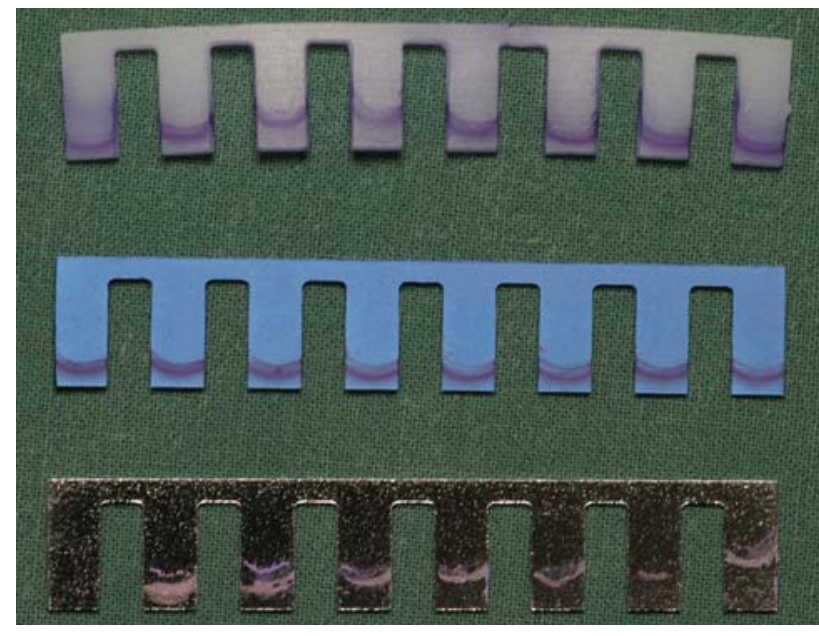

Fig. 2 This illustration shows 24-hour biofilms formed by PAO1 on all combs after staining with crystal violet.

The Netherlands) and parafilm. We left the plates for 48 hours at room temperature to allow the biofilm to elute with ethanol.

A volume of $150 \mu \mathrm{L}$ of the ethanol solution was added to a new plate and after brief, gentle shaking, the optical density (OD) in the wells was read at a wavelength of $595 \mathrm{~nm}$. The quantity of biofilm that is removed by elution cannot be expressed in percentages; however, the combs of TI and SSS seemed to be clean of CV after 48 hours of elution. The stained biofilm on PE could not be eluted entirely in ethanol (maximum time tested was 96 hours), but we standardized the time of elution to 48 hours to guarantee a reliable interpretation of the results. We tested PAO1 BF on all materials in octuplicate.

Sterile Instar-1 (I1) and Instar-3 (I3) larvae were produced by BioMonde $\mathrm{GmbH}$ (Barsbüttel, Germany). I1 maggots are freshly hatched from the egg and used after 24 hours when they measure $2 \mathrm{~mm}$ in length. I 3 maggots are full-grown larvae measuring 8 to $10 \mathrm{~mm}$ after living for 4 to 5 days. Sterile ES of Lucilia sericata were obtained by incubating $400 \mathrm{I} 1$ or $200 \mathrm{I} 3$ larvae in a $100 \mu \mathrm{L} 0.9 \%$ saline solution in sterile tubes for 1 hour at $35^{\circ} \mathrm{C}$ in darkness. Before incubation, we washed the larvae twice in PBS. After incubation, ES were removed by pipette, divided into aliquots of $200 \mu \mathrm{L}$, and stored at $-80^{\circ} \mathrm{C}$. One ES pool was collected from at least 1000 maggots, which resulted in approximately $1200 \mu \mathrm{L}$ ES per hour. We used one aliquot of the pool to determine the protein concentration using the Pierce Bicinchonic Acid Protein Assay kit (Pierce Biotechnology, Rockford, IL). The mean protein concentration of the collected ES was approximately $1500 \mu \mathrm{g} / \mathrm{mL}$ (range, $1350-1700 \mu \mathrm{g} / \mathrm{mL}$ ) and the $\mathrm{pH}$ was 8 . We tested sterility of ES in duplicate.

Three experiments were conducted on the biomaterials to answer the first research question. We examined (1) the influence of ES on an existing 24-hour biofilm to test the breakdown of BF; (2) the influence of ES on BF during a 24-hour period to test the prevention of $\mathrm{BF}$; and (3) the influence of ES on further BF of an already formed 24-hour biofilm to test inhibition and breakdown.

In Experiment 1, biofilms were allowed to grow on seven combs of each of the materials for 24 hours. Then, we rinsed the combs with distilled water and allowed them to dry. Combs were dried to guarantee the exact protein concentrations of ES in a well. One of the combs was used as a negative control and suspended in a solution of PBS. The remaining six combs were suspended in two ranges of different dilutions of ES in PBS: a low concentration range, which was $0.12,0.37,1.11,3.33,10$, and $30 \mu \mathrm{g}$ ES per well, and a high concentration range, which was $20,40,60$, 80, 100, and $120 \mu \mathrm{g}$ ES per well. The volume added per well was $25 \mu \mathrm{L}$ higher $(125 \mu \mathrm{L})$ than in the first 24-hour BF. By achieving a slightly higher liquid level in the wells, we made sure the 24-hour biofilm, which had been formed on the air-liquid interface, was in contact with ES. All wells were incubated for another 24 hours at $37^{\circ} \mathrm{C}$. Then we quantified the biofilms as described previously.

In Experiment 2, $50 \mu \mathrm{L}$ of serial dilutions of ES and PBS was dissolved in $50 \mu \mathrm{L}$ M63. We doubled the quantity of nutrient ingredients in M63 to maintain the optimal nutrient medium required for $\mathrm{BF}$ in a total volume of 100 $\mu \mathrm{L}$. The low concentrations of ES were the same as described in Experiment 1. The high range was different: 20, $30,40,50,60$, and $70 \mu \mathrm{g}$ per well. This was caused by the lower volume of ES dilutions. Sterile combs were suspended in the solutions and incubated to determine a possible inhibitory effect on BF. After 24 hours, we stained biofilms on all prongs and measured them according to the instructions described previously.

In Experiment 3 we repeated the method of Experiment 1; however, ES were now diluted in M63 instead of PBS to allow further growth of the biofilm for an additional 24 hours. To investigate the influence of ES more precisely, a more extensive range of low concentrations ( 2 to $24 \mu \mathrm{g}$ ES per well with intervals of $2 \mu \mathrm{g}$ ES per well) was tested.

In Experiment 3, we compared all values with the results of two controls: the 24-hour BF and the 48-hour BF. All measurements lower than the 24-hour biofilm were defined as a breakdown of existing biofilm caused by ES. Values lower than the 48-hour biofilm and higher than the 24-hour biofilm showed inhibition of BF. The total biofilm reduction was defined as the difference between the 48-hour biofilm and the measurement (thus reduction means inhibition and breakdown).

In every experiment, one pool of ES from I3 maggots (ES I3) with equal concentrations of ES was used. We have not compared the efficacy of ES in one experiment; however, ES were collected under strict, standard conditions by 
two of the investigators and we used only sterile ES, with $\mathrm{pH} 8$ and a protein concentration between 1350 and $1700 \mu \mathrm{g} / \mathrm{mL}$.

For the second research question, we compared the OD at $595 \mathrm{~nm}$, which reflects the $\mathrm{BF}$, among PE, TI, and SSS after 24 hours incubation of the PAO1 suspension without maggot ES.

To answer the third research question, we repeated Experiment 3 with ES from I1 maggots (ES I1) in the low concentration range to compare the effects on BF between ES I1 and ES I3. It was not possible to test the high range, because the quantity of ES that could be collected from I1 larvae was much lower than that from I3 larvae, although the protein concentration was equal in both ES.

All figures on the influence of ES on BF were log-scaled for better visualization and understanding in low and high concentrations.

To answer the last research question, we investigated the stability of the biofilm-influencing properties of maggot ES under a range of different storage conditions. Therefore, we used the method of Experiment 3 on TI combs. Nine 100$\mu \mathrm{L}$ aliquots of one batch of ES from I3 maggots were collected. Three aliquots were stored at $-80^{\circ} \mathrm{C}$, three at $+4^{\circ} \mathrm{C}$, and three at room temperature. One aliquot was taken from each storage condition after 1 day, 1 week, and 1 month. Directly incubated ES from freshly delivered maggots were tested as a control. We used a concentration of $6 \mu \mathrm{g}$ ES per well and all experiments were repeated 11 times.

The measured OD values of the biofilm experiments were converted into percentages by using the following formula:

$(\mathrm{OD}($ biofilm $) \times 100 \%) / \mathrm{OD}($ control $)$

OD (biofilm) is the BF on the prongs tested with various concentrations of ES. OD (control) is the BF after 24 hours or 48 hours on the negative control prongs. In all experiments, the 24-hour biofilm is considered $100 \%$ (in Experiment 1, it is the 24-hour biofilm after 24 hours incubation in PBS). Absolute OD values of the 24-hour biofilms on all biomaterials are reported in Results. The material-specific background absorbances were subtracted from both OD values in this formula before using it.

For the first, third, and fourth research questions, we used Student's t-test for independent groups (Table 1). In Experiments 1 and 2, for every concentration of ES separately, we compared the mean $\mathrm{BF}$ with addition of $\mathrm{ES}$ and

Table 1. Overview of the study design

Research question $\quad$ Method Statistical analysis

(1) Do sterile maggot ES break down, prevent, and/or inhibit biofilms?

(2) Does the quantity of biofilm formation depend on the material surface?

(3) Do Instar-1 and Instar-3 maggots comparably reduce biofilms?

(4) Does storage of ES under different conditions and temperature reduce the effects of ES on biofilms?
We conducted Experiment 1 to test the breakdown of biofilms by ES: ES, diluted in PBS, were added to 24hour biofilms in different concentrations.

In Experiment 2, we tested prevention of BF by ES: ES, diluted in PBS, were directly added to the nutrient medium and bacteria.

Experiment 3 repeated the method of Experiment 1 but ES were now diluted in nutrient medium to test inhibition of further biofilm growth and breakdown of existing biofilms.

All experiments were incubated during 24 hours and done in quadruplicate.

We allow 24-hour BF on comb-forming models of PE, $\mathrm{TI}$, and SSS. The experiment was done in octuplicate.

Experiment 3 was repeated with ES collected from Instar-1 maggots to compare the effects of ES I1 and ES I3 on biofilm inhibition and breakdown. The experiment was done in quadruplicate.

We stored three aliquots of one pool ES at $-80^{\circ} \mathrm{C}$, three at $+4^{\circ} \mathrm{C}$, and three at room temperature. Experiment 3 (only $6 \mu \mathrm{g}$ ES per well) was conducted on TI after 1 day, 1 week, and 1 month and ES from every storage condition was taken and biofilm inhibition and breakdown was compared. This experiment was done in 11-fold.
Student's t-test for independent variables

Experiments 1 and 2: A comparison was made between the mean BF with ES (per concentration) and the mean of the control BF without ES.

Experiment 3: A comparison was made between the mean BF with ES (per concentration) and the mean of the 24-hour BF without ES. A second comparison was made between the mean BF with ES (per concentration) and the mean of the 48-hour BF without ES.

ANOVA: A comparison was made between the quantities of biofilm on PE, TI, and SSS.

Student's t-test for independent variables

A comparison was made between the mean of the BF with ES I1 and the mean of the BF with ES I3 (per concentration).

Student's t-test for independent variables

A comparison was made between the mean $\mathrm{BF}$ with ES for every storage condition and the mean control BF without ES.

$\mathrm{BF}=$ biofilm formation; $\mathrm{PE}=$ polyethylene; $\mathrm{TI}=$ titanium; $\mathrm{SSS}=$ surgical stainless steel; $\mathrm{ES}=$ excretions and secretions; PBS = phosphatebuffered saline; ES I1 = ES from Instar 1 maggots; ES I3 = ES from Instar 3 maggots; ANOVA = analysis of variance. 
the mean BF without addition of ES. In Experiment 3, the mean $\mathrm{BF}$ of the samples with ES was compared with the mean BF of controls after 24 hours and 48 hours. For the third research question, we compared the mean BF with ES I1 and the mean BF with ES I3 for each concentration separately. The last research question was analyzed in the same way as Experiment 3, comparing the mean BF with ES for every storage condition with the mean control BF without ES. For the second research question, we used oneway ANOVA followed by the least significant difference post hoc test to analyze if the quantity of BF depended on the material surface. Therefore, we compared the quantity of biofilm among PE, TI, and SSS. We performed all statistical analyses using SPSS ${ }^{\circledR}$ for Windows ${ }^{\circledR}$, Version 11.0 (SPSS Inc, Chicago, IL).

\section{Results}

Experiment 1 showed the addition of ES to existing biofilms decreased these biofilms compared with biofilms without addition of ES for TI and PE. In Experiment 1, the breakdown (all $\mathrm{p}<0.001$ ) on TI was maximal $(58 \%)$ in the range between 0.37 and $10 \mu \mathrm{g}$ ES per well so there was less biofilm compared with the control (Fig. 3A). For PE, Experiment 1 showed a maximal breakdown (all $\mathrm{p}<0.001)$ of $82 \%$ in the range between 1 and $10 \mu \mathrm{g}$ ES per well (Fig. 3B). BF for all concentrations was decreased compared with the control. Prevention of biofilm was

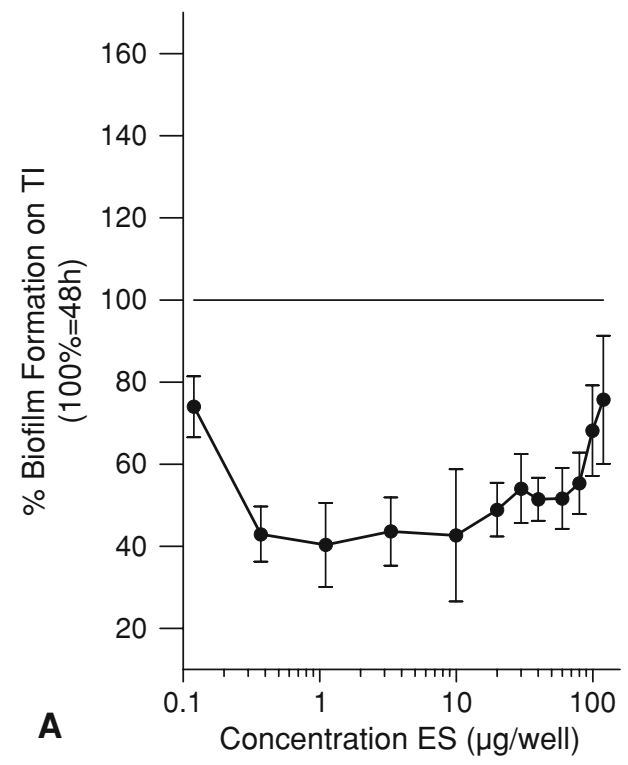

Fig. 3A-B These figures show a comparison between the mean of existing biofilms without addition of ES and with addition of ES and answer partially the first research question, whether ES break down biofilms. The control 48-hour biofilm is defined as $100 \%$ and all values lower than this show breakdown of biofilm by ES. (A) Biofilm tested in Experiment 2. BF was reduced (all p $<0.001$ ) compared with control without ES on TI between 10 and $30 \mu \mathrm{g}$ ES per well (Fig. 4A). On PE, BF was less (all $\mathrm{p} \leq 0.013$ ) for all concentrations between 0.37 and $30 \mu \mathrm{g}$ ES per well compared with the control BF (Fig. 4B). Inhibition of further biofilm growth and breakdown by ES was tested in Experiment 3. Addition of ES to existing biofilms resulted in less $\mathrm{BF}$ compared with $\mathrm{BF}$ without ES. ES further inhibited (all $p \leq 0.038$ ) biofilm on TI in a concentration range between 2 and $18 \mu \mathrm{g}$ ES per well to a minimum of $47 \%$ of its original size (without ES) (Fig. 5A). Experiment 3 also showed breakdown (all $\mathrm{p} \leq 0.028$ ) of biofilm on TI with a maximum of $53 \%$ in the range between 2 and $6 \mu \mathrm{g}$ ES per well (Fig. 5A). On PE, the biofilm was inhibited to a minimum of $37 \%$ at concentrations of 20 and $22 \mu \mathrm{g}$ ES per well (both $\mathrm{p}<0.001$; Fig. 5B). Less (all $p \leq 0.018$ ) BF with ES was seen for all concentrations greater than $6 \mu \mathrm{g}$ ES per well. Breakdown of PE in Experiment 3 showed reduced ( $p<0.001$ for both concentrations) biofilm concentrations of $63 \%$ for 20 and $22 \mu \mathrm{g}$ ES per well compared with the controls (Fig. 5B).

$\mathrm{BF}$ was greater on $\mathrm{PE}\left(\mathrm{OD}_{595} 0.381\right.$ after background absorbances subtracted $)$ than on TI $\left(\mathrm{p}<0.001 ; \mathrm{OD}_{595}\right.$ $0.153)$ and SSS $\left(\mathrm{p}<0.001 ; \mathrm{OD}_{595}\right.$ 0.051) (Fig. 6; Table 2).

On TI, biofilm reduction was greater $(\mathrm{p}=0.022)$ by ES I3 than by ES I1 at $16 \mu \mathrm{g}$ ES per well and all concentrations higher (all $\mathrm{p} \leq 0.001$ ) than $20 \mu \mathrm{g}$ ES per well (Fig. 7A). On PE, biofilm reduction also was greater (all

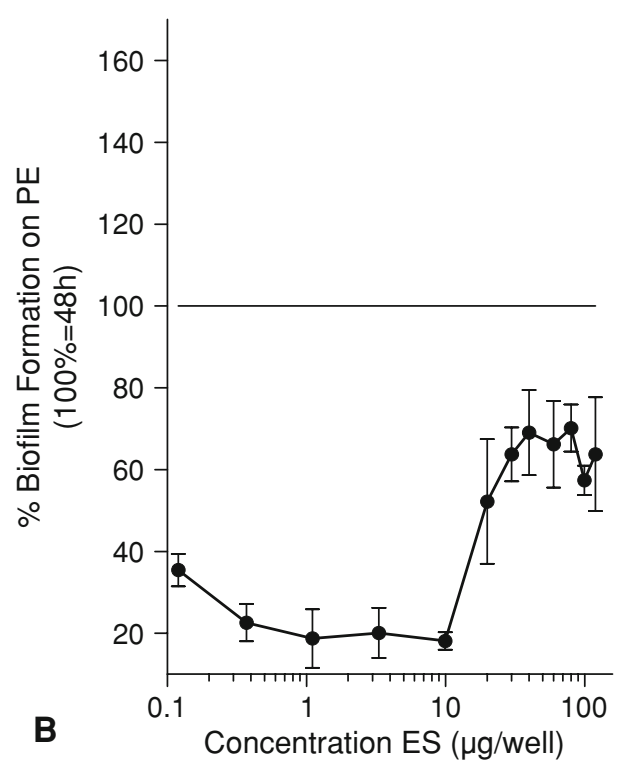

was broken down (all p values $<0.001$ ) on TI by ES in a concentration range from 0.37 to $80 \mu \mathrm{g}$ ES per well. (B) Biofilm was broken down (all p values $<0.001$ ) on PE by ES in a concentration range from 0.12 to $10 \mu \mathrm{g}$ ES per well. ES = excretions and secretions; $\mathrm{TI}=$ titanium; $\mathrm{PE}=$ polyethylene. 


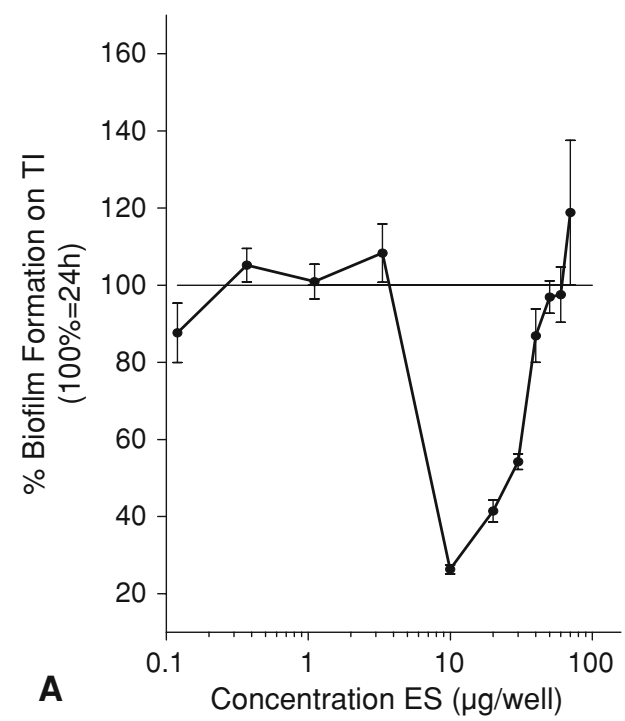

Fig. 4A-B These figures show a comparison between the mean BF with ES that were directly added to the bacteria and the mean control 24-hour BF without ES (defined as 100\%) and answer partially the first research question, whether ES prevent BF. All values lower than $100 \%$ show prevention of BF. (A) Biofilm was prevented (all p

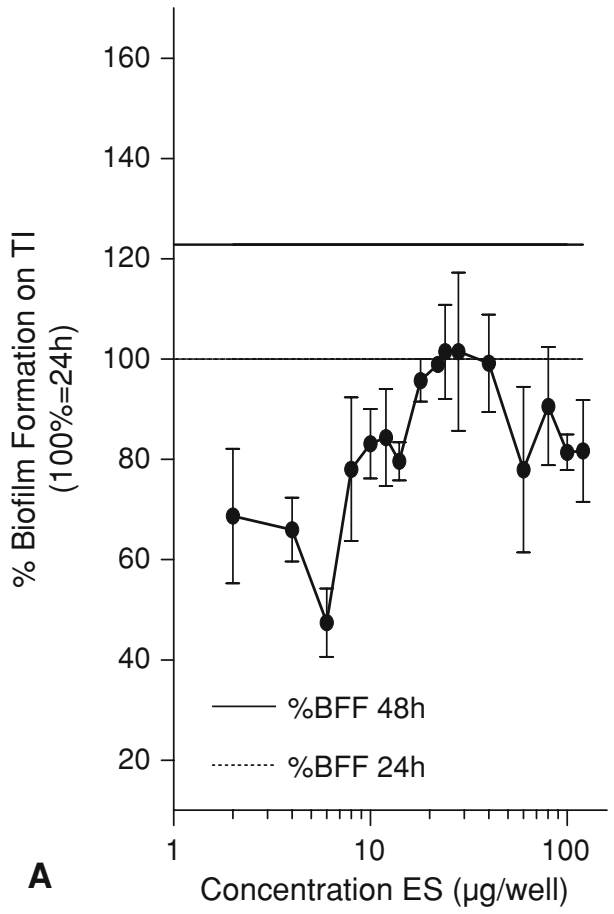

Fig. 5A-B These figures show a comparison between the mean BF with ES and the mean control 24-hour BF without ES (defined as $100 \%$ ) and answer partially the first research question, whether ES break down existing biofilms. All values lower than $100 \%$ show breakdown of biofilm by ES. These figures also show a comparison between the mean $\mathrm{BF}$ with $\mathrm{ES}$ and the mean control 48-hour BF without ES (122\% on TI and $163 \%$ on PE) and answer partially the first research question, whether ES inhibit further biofilm growth. All values lower than the 48-hour biofilm show inhibition of further

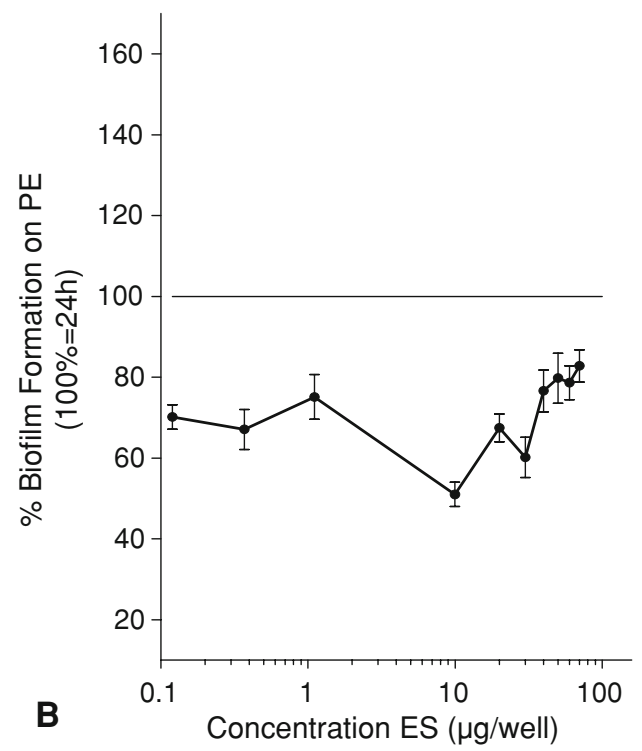

values $<0.001)$ on TI by ES in a concentration range from 10 to 30 $\mu \mathrm{g}$ ES per well. (B) Biofilm was prevented (all p values $\leq 0.013$ ) on $\mathrm{PE}$ by ES in a concentration range from 0.12 to $30 \mu \mathrm{g}$ ES per well. $\mathrm{BF}=$ biofilm formation; $\mathrm{ES}=$ excretions and secretions; $\mathrm{TI}=$ titanium; $\mathrm{PE}=$ polyethylene.

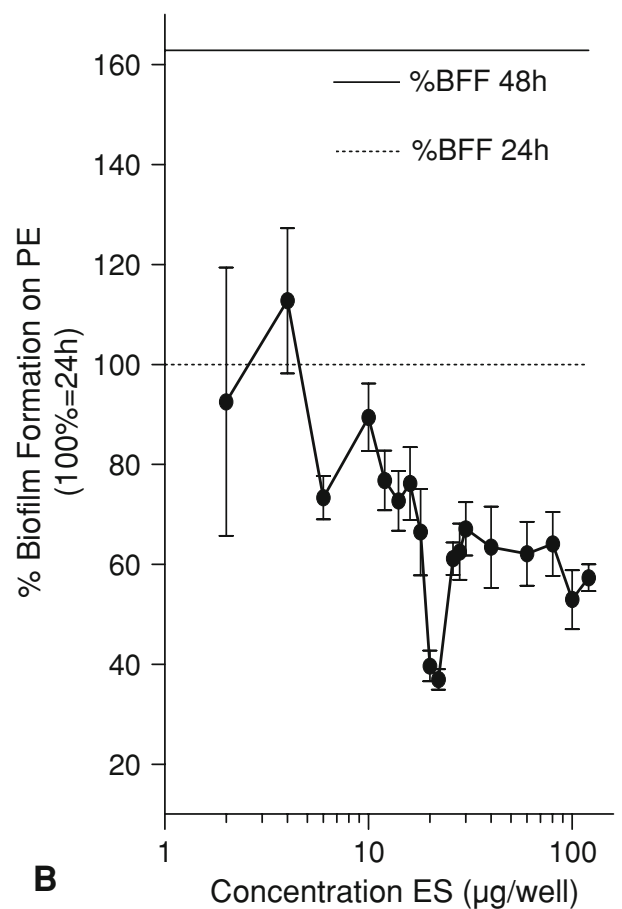

BF. (A) Biofilm was broken down (all p values $\leq 0.028$ ) on TI by ES in a concentration range from 2 to $6 \mu \mathrm{g}$ ES per well. Inhibition (all $\mathrm{p}$ values $\leq 0.038$ ) occurred in a concentration range from 2 to 18 $\mu \mathrm{g}$ ES per well. (B) Biofilm was broken down (all p values $\leq 0.011$ ) on PE by ES in a concentration range of $20 \mu \mathrm{g}$ ES per well or greater. Inhibition (all $\mathrm{p}$ values $\leq 0.018$ ) is observed in a concentration range of $6 \mu \mathrm{g}$ ES per well or greater. $\mathrm{BFF}=$ biofilm formation; $\mathrm{ES}=$ excretions and secretions; $\mathrm{TI}=$ titanium; $\mathrm{PE}=$ polyethylene. 


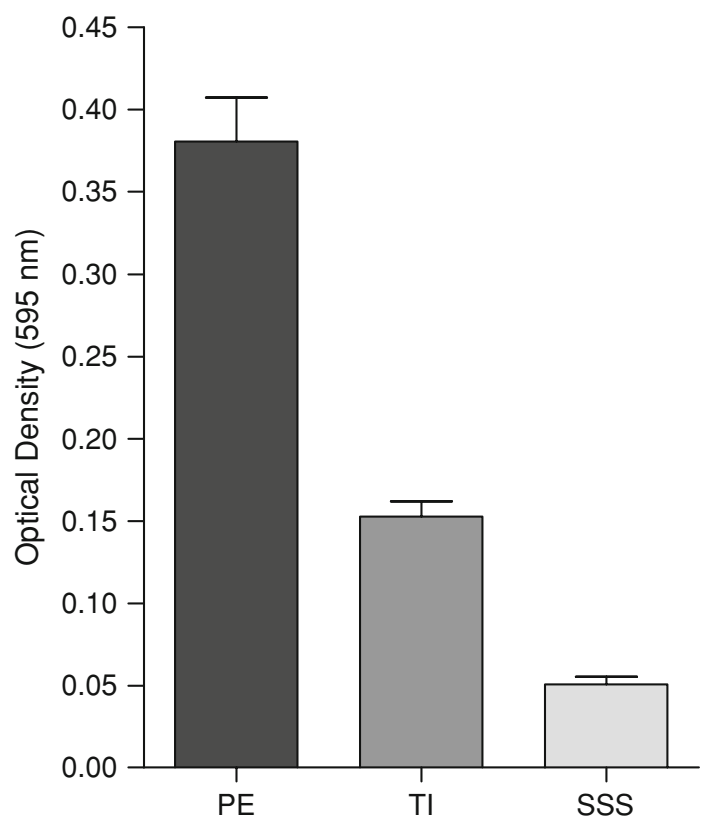

Fig. 6 The optical densities reflect the quantities of biofilms formed in 24 hours on PE, TI, and SSS and answer the second research question, whether $\mathrm{BF}$ depend on the material surface. PE has the highest BF followed by TI and SSS with the weakest attachment to biofilm. $\mathrm{PE}=$ polyethylene; $\mathrm{TI}=$ titanium; $\mathrm{SSS}=$ surgical stainless steel; $\mathrm{BF}=$ biofilm formation.

Table 2. Optical density $(595 \mathrm{~nm})$ for a 24-hour biofilm and a 48-hour biofilm on all materials

\begin{tabular}{llll}
\hline $\begin{array}{l}\text { Optical density } \\
(595 \mathrm{~nm})\end{array}$ & $\begin{array}{l}\text { 24-hour } \\
\text { biofilm }\end{array}$ & $\begin{array}{l}\text { 48-hour } \\
\text { biofilm }\end{array}$ & $\begin{array}{l}\text { Background } \\
\text { absorbance }\end{array}$ \\
\hline Polyethylene & 0.381 & 0.620 & 0.0924 \\
Titanium & 0.153 & 0.187 & 0.0520 \\
Surgical stainless steel & 0.051 & 0.099 & 0.0371 \\
\hline
\end{tabular}

$\mathrm{p} \leq 0.039$ ) by ES I3 than by ES I1, but in the concentration range from 0.37 to $14 \mu \mathrm{g}$ ES per well (Fig. 7B).

All stored ES inhibited biofilm $(\mathrm{p}=0.010)$ compared with the control; even ES stored at room temperature for 1 month showed 18\% inhibition (Fig. 8). The ES with the best biofilm reduction properties were directly used ES, showing $45 \%$ inhibition $(\mathrm{p}<0.001)$ of biofilm compared with control.

\section{Discussion}

$\mathrm{BF}$ on orthopaedic biomaterials causes severe infections that are very difficult to treat and often lead to invalidating consequences for patients $[6,19,32]$. Therefore, it is important to investigate new treatment possibilities for these kind of infections. In clinical practice, we observed the successful effects of sterile maggots captured in permeable bags on severely infected wounds that were suspected for $\mathrm{BF}$ and therefore, we hypothesized sterile maggot ES would reduce BF. We also asked whether the quantity of biofilms depended on the biomaterial surface, whether ES from Instar-1 maggots and Instar-3 maggots comparably reduced biofilms, and whether storage of ES under different conditions and temperature decreased the effects of ES on BF.

We note several limitations of our study. We tested only the BF of P. aeruginosa. Although P. aeruginosa is not the most common cause of infection, it is difficult to treat when present. Moreover, P. aeruginosa (PAO1 strain) can form strong biofilms in vitro and therefore is used as a model in our experiments for 48 hours maximum [21]. However, these biofilms will not be fully mature, but basic information regarding influence of maggot ES against the $P$. aeruginosa biofilm can be obtained [23]. We used the crystal violet assay as a standard method to quantify biofilms, but other methods could be used in vitro [36] and in vivo [12]. We attempted to adapt our study conditions to those in clinical practice by using biomaterials produced according to specifications for common patient implants. Our ES were collected under standard conditions in darkness, had an acidity of $\mathrm{pH} 8$, and were incubated at a temperature of $35^{\circ} \mathrm{C}$. Therefore, we believe these results are representative and that maggot ES have a strong capacity to break down existing biofilms and inhibit their formation.

Our findings suggest BF on TI and PE is successfully prevented and inhibited by sterile maggot ES and biofilms on both materials are broken down by ES. Furthermore, the quantity of BF depends on the biomaterial surface and SSS is the best biomaterial in its physiologic behavior with respect to $\mathrm{BF}$ by $\mathrm{P}$. aeruginosa. We found ES I3 are more capable of reducing BF than ES I1 and sterile maggot ES are still effective after storing it for 1 month at room temperature.

The use of maggot therapy for wound débridement is an ancient method that is widely used for treatment of acute and chronic wound infections in trauma and orthopaedic patients, although the exact mechanism of action of the maggots in the healing process is unknown. Some clinical reports document successful results of maggots in the débridement, disinfection, and healing of many types of severely infected wounds that failed to heal with conventional treatment $[11,27,30,35]$. Research of débridement mechanisms underlying MDT show the production of proteolytic and chymotrypsin-like enzymes in ES, which could degrade extracellular matrix components in wounds $[3,4,33]$. A more specific study reported the influence of ES on the behavior of fibroblasts on extracellular matrix surfaces; ES could modulate the adhesion and spreading of 
Fig. 7A-B These figures show a comparison between the mean of the BF with ES I1 and the mean of the BF with ES I3 and answer the third research question, whether ES I1 and ES I3 comparably reduce $\mathrm{BF}$. The comparison is made for every concentration separately. (A) The biofilm reduction on TI is greater (all $\mathrm{p}$ values $\leq 0.022$ ) with ES I3 than with ES I1 in a concentration range of $20 \mu \mathrm{g}$ ES per well or greater. (B) The biofilm reduction on $\mathrm{PE}$ is greater $($ all $\mathrm{p}$ values $\leq 0.039$ ) with ES I3 than with ES I1 in a concentration range of $14 \mu \mathrm{g}$ ES per well or less. ES = excretions and secretions; $\mathrm{TI}=$ titanium; $\mathrm{PE}=$ polyethylene $; \quad \mathrm{BF}=$ biofilm formation.
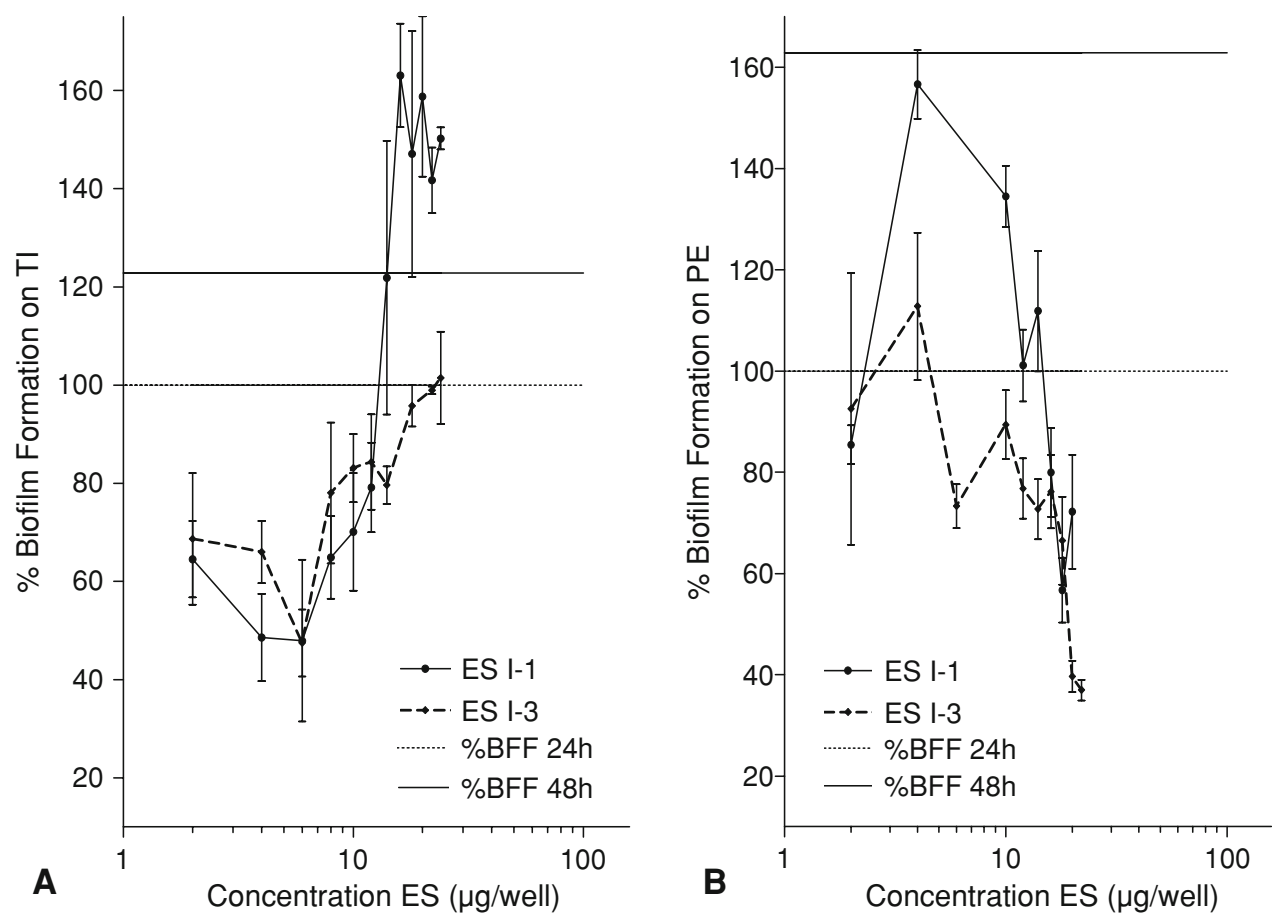

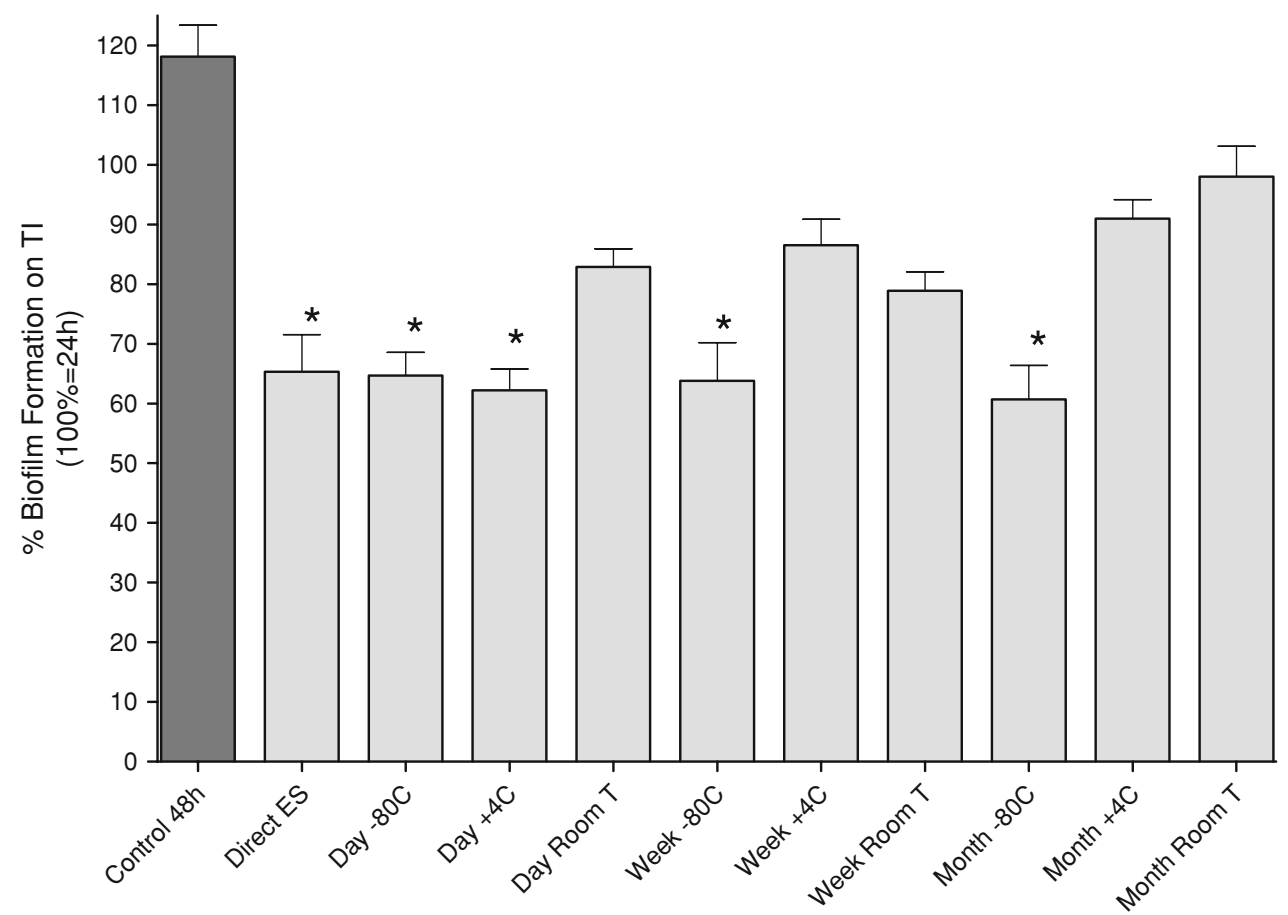

Fig. 8 This figure shows the comparison between the mean of the samples of BF with ES for every storage condition with the mean control BF without ES and answers the fourth research question, whether storage of ES under different conditions and temperatures reduces the effects of ES on BF. Direct ES were immediately incubated ES from freshly delivered I3 maggots. A concentration of $6 \mu \mathrm{g}$ ES per well was used and added to an existing 24-hour biofilm on TI (defined as 100\%). All values lower than $100 \%$ show breakdown of biofilm and all values lower than the 48-hour biofilm without ES (118\%) show inhibition of BF. All storage conditions inhibit (for all conditions, $p$ values $\leq 0.010$ ) BF. "These are the storage conditions that showed a breakdown (for these conditions, $\mathrm{p}$ values $\leq 0.041)$ of biofilm. ES $=$ excretions and secretions; $\mathrm{TI}=$ titanium. 
fibroblasts on extracellular matrix, stimulate proliferation and migration of fibroblasts, and therefore enhance tissue formation and accelerate healing [10]. In another study, the mechanical action of maggots has been suggested in aiding tissue débridement [17]. One of the most intriguing hypotheses regarding the effectiveness of maggots in infected wounds is the idea that Lucilia sericata ES have antibacterial properties. Several studies have reported in vitro antibacterial activity, but not with consistent results. Kerridge et al. performed a zone of inhibition assay and found inhibition of bacterial growth by ES against Staphylococcus aureus and P. aeruginosa [13]. Bexfield et al., who performed the same zone of inhibition assay, did not find this inhibition or decrease in bacterial growth [2]. However, they found inhibition in the turbidimetric assay and concluded the antibacterial activity depends on the kind of experiment. The first report that described the possible activity of ES against biofilms of $\mathrm{P}$. aeruginosa and $\mathrm{S}$. aureus in in vitro experiments is the recently published paper by van der Plas et al. [22].

Our results showed the physical properties of the biomaterials influence the strength of BF. Given the very low rate of BF, SSS seemed the best biomaterial in vitro. $\mathrm{P}$. aeruginosa biofilms attached strong to TI in our study, according to Staphylococcus biofilms, as described by Harris et al. [9]. Furthermore, in our study ES I3 resulted in a greater reduction of the biofilm than ES I1, although the protein concentration is equal in both ES. We presume the composition of ES I1 differs from that of ES I3. I3 larvae produce much more ES than I1 larvae. This may explain why ES I3 are more effective in reducing biofilm. They also would allow obtaining and preparing large quantities. We have not tested the effectivity between the pools of ES I3; however, we have a few points in which we can compare the different pools. The 48-hour biofilm on TI, for example, is approximately $122 \%$ in Experiments $5 \mathrm{a}$ and $7 \mathrm{a}$ and $118 \%$ in Experiment 8 . At $6 \mu \mathrm{g}$ ES per well, the biofilm on TI was reduced to $47 \%$ of its original size in Experiments 5a and $7 \mathrm{a}$ and $64 \%$ in Experiment 8 . We expect the variability is not large because of the strict conditions that were maintained during collection, as described before.

Our temperature tolerance experiment shows in clinical practice, we could store maggot ES at room temperature for a few days without apparent loss of quality.

Protein concentrations of ES appeared optimal for biofilm reduction in concentrations up to 10 to $20 \mu \mathrm{g}$ ES per well (80 to $160 \mu \mathrm{g} \mathrm{ES} / \mathrm{mL}$ ). van der Plas et al. also reported biofilm reduction at concentrations up to $20 \mu \mathrm{g}$ ES per well [22]. In our in vitro experiments, each maggot produced more than $1.8 \mu \mathrm{g}$ ES per hour. If we assume the I3 larvae have the same protein production every hour, it can be derived that two maggots per square centimeter biofilm in wounds during 24 hours are sufficient to reduce the biofilm. This is much lower than the maggot quantities (from five to ten larvae per square centimeter wound surface) that are recommended for débridement [31]. Although still active, higher concentrations provided less biofilm degradation. To reduce $\mathrm{BF}$ on $\mathrm{PE}$, higher concentrations of $\mathrm{ES}$ were needed than on TI. This may be the result of the more porous structure of PE, resulting in tighter adherence of biofilm. There are a few possible explanations for the fact that the efficacy of ES at higher concentrations (greater than $20 \mu \mathrm{g}$ ES per well) decreases. At higher enzyme concentrations, acidity of the environment changes faster than at lower concentrations and this change can play a role. We suggest a cofactor needed to facilitate the process is depleted or an enzyme with a negative allosteric effect is released. Another explanation could be a covalent modification disturbs the process and stops it or that membranes in the biofilm are damaged and permeate an enzyme that inhibits the reduction [16]. Further research is needed to explore the interaction of ES and BF.

We believe this work may be relevant to patient care, because biofilms make treatment of infected orthopaedic implants and prostheses more difficult [6, 19, 32]. For the future, the influence of sterile ES on BF by different bacterial species on orthopaedic implants is our current topic of research.

Acknowledgments We thank Dr. Saskia le Cessie from the Department of Medical Statistics and Bioinformatics (Leiden University Medical Center) for her contribution and advice in analyzing our statistical results.

\section{References}

1. Baer WS. The treatment of chronic osteomyelitis with the maggot (larvae of the blowfly). J Bone Joint Surg. 1931;13:438-475.

2. Bexfield A, Nigam Y, Thomas S, Ratcliffe NA. Detection and partial characterisation of two antibacterial factors from the excretions/secretions of the medicinal maggot Lucilia sericata and their activity against methicillin-resistant Staphylococcus aureus (MRSA). Microbes Infect. 2004;14:1297-1304.

3. Casu RE, Pearson RD, Jarmey JM, Cadogan LC, Riding GA, Tellam RL. Extretory/secretory chymotrypsin from Lucilia cuprina: purification enzymatic specificity and amino acid sequence deduced from mRNA. Insect Mol Biol. 1994;3:201-211.

4. Chambers L, Woodrow S, Brown AP, Harris PD, Philips D, Hall M, Church JC, Pritchard DI. Degradation of extracellular matrix components by defined proteinases from the greenbottle larva Lucilia sericata used for the clinical debridement of non-healing wounds. Br J Dermatol. 2003;148:14-23.

5. Costerton JW. Introduction to biofilm. Int J Antimicrob Agents. 1999;11:217-221.

6. Costerton JW. Biofilm theory can guide the treatment of devicerelated orthopaedic infections. Clin Orthop Relat Res. 2005;437: 7-11.

7. Costerton JW, Stewart PS, Greenberg EP. Bacterial biofilms: a common cause of persistent infections. Science. 1999;284:1318 1322. 
8. Hall-Stoodley L, Costerton JW, Stoodley P. Bacterial biofilm: from the natural environment to infectious diseases. Nat Rev Microbiol. 2004;2:95-108.

9. Harris LG, Meredith DO, Eschbach L, Richards RG. Staphylococcus aureus adhesion to standard micro-rough and electropolished implant materials. J Mater Sci Mater Med. 2007; 18:1151-1156.

10. Horobin AJ, Shakesheff KM, Pritchard DI. Promotion of human dermal fibroblast migration, matrix remodelling and modification of fibroblast morphology within a novel 3D model by Lucilia sericata larval secretions. J Invest Dermatol. 2006;126:1410-1418.

11. Jukema GN, Menon AG, Bernards AT, Steenvoorde P, Taheri Rastegar A, Van Dissel JT. Amputation-sparing treatment by nature: 'surgical' maggots revisited. Clin Infect Dis. 2002;35: $1566-1571$

12. Kadurugamuwa JL, Francis KP. Bioluminescent imaging of bacterial biofilm infections in vivo. Methods Mol Biol. 2008;431: 225-239.

13. Kerridge A, Lappin-Scott H, Stevens JR. Antibacterial properties of larval secretions of the blowfly, Lucilia sericata. Med Vet Entomol. 2005;3:333-337.

14. King EO, Ward MK, Raney DE. Two simple media for the demonstration of pyocyanin and fluorescein. J Lab Clin Med. 1954:44:301-307.

15. Levy SB. The challenge of antibiotic resistance. Scientific American. 1998;278:46-53.

16. Meisenberg G, Simmons WH. Principles of Medical Biochemistry. St Louis, MO: Mosby; 1998.

17. Mumcuoglu K, Miller J, Mumcuoglu M, Friger F, Tarshis M. Destruction of bacteria in the digestive tract of the maggot of Lucilia sericata (Diptera: Calliphoridae). J Med Entomol. 2001;38:161-166.

18. Nablo BJ, Prichard HL, Butler RD, Klitzman B, Schoenfisch MH. Inhibition of implant-associated infections via nitric oxide release. Biomaterials. 2005;26:6984-6990.

19. Neut D, Hendriks JG, van Horn JR, van der Mei HC, Busscher HJ. Pseudomonas aeruginosa biofilm formation and slime excretion on antibiotic loaded bone cement. Acta Orthop. 2005;76:109-114.

20. O'Toole GA. To build a biofilm. J Bacteriol. 2003;185:26872689.

21. O'Toole GA, Kolter R. Initiation of biofilm formation in Pseudomonas fluorescens WCS365 proceeds via multiple, convergent signalling pathways: a genetic analysis. Mol Microbiol. 1998;28:449-461.

22. van der Plas MJ, Jukema GN, Wai SW, Dogterom-Ballering HC, Lagendijk EL, van Gulpen C, van Dissel JT, Bloemberg GV, Nibbering $\mathrm{PH}$. Maggot excretions/secretions are differentially effective against biofilm of Staphylococcus aureus and Pseudomonas aeruginosa. J Antimicrob Chemother. 2008;61:117-122.

23. Sauer K, Camper AK, Ehrlich GD, Costerton JW, Davies DG. Pseudomonas aeruginosa displays multiple phenotypes during development as a biofilm. J Bacteriol. 2002;184:1140-1154.

24. Sherman RA, Hall MJR, Thomas S. Medicinal maggots: an ancient remedy for some contemporary afflictions. Аnnu Rev Entomol. 2000;45:55-81.

25. Sherman RA, Pechter EA. Maggot therapy: a review of the therapeutic applications of fly larvae in human medicine, especially for treating osteomyelitis. Med Vet Entomol. 1988;2:225230.

26. Sritharan M, Shritharan V. Emerging problems in the management of infectious diseases: the biofilms. Indian J Med Microbiol. 2004;22:140-142.

27. Steenvoorde P, Jukema GN. The antimicrobial activity of maggots: in-vivo results. J Tissue Viability. 2004;14:97-101.

28. Stewart PS, Costerton JW. Antibiotic resistance of bacteria in biofilms. Lancet. 2001;358:135-138.

29. Stover CK, Pham XQ, Erwin AL, Mizoguchi SD, Warrener P, Hickey MJ, Brinkman FS, Hufnagle WO, Kowalik DJ, Lagrou M, Garber RL, Goltry L, Tolentino E, Westbrock-Wadman S, Yuan Y, Brody LL, Coulter SN, Folger KR, Kas A, Larbig K, Lim R, Smith K, Spencer D, Wong GK, Wu Z, Paulsen IT, Reizer J, Saier MH, Hancock RE, Lory S, Olson MV. Complete genome sequence of Pseudomonas PAO1, an opportunistic pathogen. Nature. 2000;406:947-948.

30. Tanyuksel M, Araz E, Dundar K, Uzun G, Gumus T, Alten B, Saylam F, Taylan-Ozkan A, Mumcuoglu KY. Maggot debridement therapy in the treatment of chronic wounds in a military hospital setup in Turkey. Dermatology. 2005;210:115-118.

31. Thomas S, Jones M, Shutler S, Andrews A. Wound care: all you need to know about maggots. Nurs Times. 1996;92:63-70.

32. Trampuz A, Widmer AF. Infections associated with orthopedic implants. Curr Opin Infect Dis. 2006;19:349-356.

33. Vistnes L, Lee R, Ksander A. Proteolytic activity of blowfly larvae secretions in experimental burns. Surgery. 1981;90:835841.

34. Wainwright M. Miracle Cure: The Story of Penicillin and the Golden Age of Antibiotics. Oxford, UK: Basil Blackwell; 1990.

35. Wang J, Wang S, Zhao G, Wang Z, Lineaweaver WC, Zhang F. Treatment of infected wounds with maggot therapy after replantation. J Reconstr Microsurg. 2006;22:277-280.

36. Woodworth BA, Tamashiro E, Bhargave G, Cohen NA, Palmer $\mathrm{JN}$. An in vitro model of Pseudomonas aeruginosa biofilms on viable airway epithelial cell monolayers. Am J Rhinol. 2008;22:235-238. 